K. AZUKAWA

KODAI MATH. J.

8 (1985), $79-89$

\title{
AN INTRINSIC FIBRE METRIC ON THE $n$-TH SYMMETRIC TENSOR POWER OF THE TANGENT BUNDLE
}

\author{
By Kazuo AzUKawa
}

0. Introduction. Let $H(M)$ be the Hilbert space consisting of all squareintegrable holomorphic $m$-forms on an $m$-dimensional complex manifold $M$. The Bergman form $K$ is defined as a specific holomorphic $2 m$-form on the product manifold $M \times \bar{M}$, where $\bar{M}$ is the conjugate complex manifold of $M$. Let $z=$ $\left(z^{1}, \cdots, z^{m}\right)$ be a coordinate system with defining domain $U_{z}$, and $k_{z}$ be the Bergman function relative to $z$, i.e. $K(p, \bar{p})=k_{z}(p)\left(d z^{1} \wedge \cdots \wedge d z^{m}\right)_{p} \wedge\left(d \bar{z}^{1} \wedge \cdots \wedge d \bar{z}^{m}\right)_{\bar{p}}$, $p \in U_{z}$. In general, $k_{z} \geqq 0$. In Kobayashi [4], the following conditions are considered :

(A.1) For every $p \in M$, there exists $\alpha \in H(M)$ such that $\alpha(p) \neq 0$.

(A.2) For every non-zero tangent vector $X$ at $p \in M$, there exists $\alpha \in H(M)$ such that $\alpha(p)=0$ and $X . \alpha(p) \neq 0$.

Suppose (A.1) holds. Then $k_{z}>0$ for every $z$, and the Bergman pseudo-metric $g$, with components $g_{a \bar{b}}=\partial_{a} \overline{\partial_{b}} \cdot \log k_{z}$, is defined. Furthermore, the following is known $([4])$ :

$\left(\mathrm{K}_{1}\right) \quad g$ is a metric if and only if (A.2) holds.

If $M$ satisfies (A.1) and (A.2), and if $R_{a \bar{c} c \bar{d}}$ are the components of the hermitian curvature tensor of the Bergman metric, then the following are known ([4]):

$\left(\mathrm{K}_{2}\right)$ Set $\hat{R}_{a c \bar{c} \bar{d}}=R_{a \bar{c} c \bar{d}}+g_{a \bar{b}} g_{c \bar{d}}+g_{a \bar{d}} g_{c \bar{c}}$. Then $\sum \bar{R}_{a c \bar{b} \bar{v}} v^{a} v^{c} \bar{v}^{b} \bar{v}^{d} \geqq 0$ for every $\left(v^{1}, \cdots, v^{m}\right) \in \boldsymbol{C}^{m}$.

$\left(\mathrm{K}_{3}\right) \quad \hat{R}_{a c \bar{b} \bar{d}}=k^{-1}\left(k_{a c \overline{b \bar{d}}}-k^{-1} k_{a c} k_{\overline{b \bar{d}}}\right)-k^{-2} \sum g^{\bar{t} s}\left(k_{a c \bar{t}}-k^{-1} k_{a c} k_{\bar{t}}\right)\left(k_{s \overline{b \bar{d}}}-k^{-1} k_{\overline{b \bar{d}}} k_{s}\right)$, where $k=k_{z}, k_{a c}=\partial_{a} \partial_{c} . k$, etc., and $\left(g^{\bar{t} s}\right)=\left(g_{a \bar{b}}\right)^{-1}$.

In the preceding joint paper [2] with Burbea, conditions $\left(C_{n}\right)$ are defined so that $\left(C_{0}\right)$ (resp. $\left(C_{1}\right)$ ) coincides with (A.1) (resp. (A.2)). Furthermore, under assumption $\left(C_{0}\right)$, non-negative functions $\mu_{0, n}$, which are biholomorphic invariants, on the tangent bundle are introduced.

In the present paper, we first note (Proposition 1.2) that the functions $\mu_{0, n}$ on the tangent bundle are, in general, upper semi-continuous, and show (Theorem 2.1) that when $M$ satisfies condition $\left(C_{0}\right)$ there exists a unique fibre pseudometric $g^{(n)}$ on the $n$-th symmetric tensor power $S^{n} T(M)$ of the tangent bundle

Received March 15, 1984 
$T(M)$ for $n \in N$ such that

$$
(n !)^{-2} \mu_{0, n}(X)=g^{(n)}\left(X^{n}, \bar{X}^{n}\right), \quad X \in T(M) ;
$$

in particular, the pseudo-metric $g^{(1)}$ coincides with the Bergman one stated before. In addition, if $M$ satisfies also $\left(C_{1}\right), \cdots,\left(C_{n-1}\right)$, then $g^{(n)}$ is differentiable (Theorem 2.5), and assertion $\left(K_{1}\right)$ is generalized as follows (Theorem 2.6): $g^{(n)}$ is a metric if and only if $\left(C_{n}\right)$ holds. Finally, we consider the curvature of the hermitian connection of the hermitian vector bundle $\left(S^{n} T(M), g^{(n)}\right)$ in the sense of Kobayashi and Nomizu [6]. In view of Fuks [3], the component $g_{a b c \bar{c}}^{(2)}$ coincides with $\hat{R}_{a b \overline{c d}} / 4$ given in $\left(\mathrm{K}_{2}\right)$, and $\left(\mathrm{K}_{2}\right)$ gives a relationship between the curvature of $g^{(1)}$ and the metric $g^{(2)}$. We generalize this relationship to the one between the curvature of $g^{(n)}$ and the metric $g^{(n+1)}$ (Theorem 3.1). The proof of Theorem 3.1 is done by observing formula $\left(\mathrm{K}_{3}\right)$ and by the use of a recurrence formula (Proposition 3.5) for the components of $g^{(n)}$.

1. Preliminaries. Throughout this paper, we are concerned with a fixed paracompact connected complex manifold $M$ of dimension $m$. The term "coordinate $z$ " stands for a local holomorphic coordinate system $z=\left(z^{1}, \cdots, z^{m}\right)$ of $M$ with defining domain $U_{z}$. For simplicity, we set $\partial_{a}^{z}=\partial / \partial z^{a}(a=1, \cdots, m)$, and $d z=d z^{1} \wedge \cdots \wedge d z^{m}$. For a multi-index $A=\left(a_{1}, \cdots, a_{n}\right) \in \mathrm{MI}(n)=\{1, \cdots, m\}^{n}$, set $\partial_{A}^{z}=\partial_{a_{1}}^{z} \cdots \partial_{a_{n}}^{z}$. In particular, $\operatorname{MI}(0)=\{\phi\}$, and $\partial_{\phi}^{z}$ means the identity operator acting on functions on $U_{z}$. For a constant vector $v=\left(v^{1}, \cdots, v^{m}\right)$ in $\boldsymbol{C}^{m}$, set $\partial_{v}^{z}=\sum_{a=1}^{m} v^{a} \partial_{a}^{z}$. The powers $\left(\partial_{v}^{z}\right)^{n}(n=0,1, \cdots)$ are naturally defined. We denote by $\bar{M}$ the conjugate complex manifold of $M$, and denote by $\rho: M \ni p \mapsto \bar{p} \in \bar{M}$ the conjugation. For a coordinate $z$ with defining domain $U_{z}$, we denote by $\bar{z}$ the conjugate coordinate of $z$ with defining domain $\overline{U_{z}}$, i. e. $\bar{z}(\bar{p})=\overline{z(p)}$ for $p \in U_{z}$.

We denote by $H(M)$ the separable Hilbert space consisting of all holomorphic $m$-forms $\alpha$ on $M$ which satisfy $\|\alpha\|^{2}=\left(\sqrt{-1} m^{2} / 2^{m}\right) \int_{M} \alpha \wedge \bar{\alpha}<+\infty$, and denote by (, ) the hermitian inner product on $H(M)$ corresponding to the norm $\|\cdot\|$. There exists a unique $(2 m, 0)$-form $K$, called the Bergman form, on the product manifold $M \times \bar{M}$ such that $K(\cdot, \bar{p}) / d \bar{z}_{\bar{p}} \in H(M)$ and $\alpha(p) / d z_{p}=\left(\alpha, K(\cdot, \bar{p}) / d \bar{z}_{\bar{p}}\right)$ for every $p \in M$ and $\alpha \in H(M)$, where $z$ is a coordinate around $p$ (cf., e.g., [2 ; Corollary 2.6]). Thus, $\left(1_{M}, \rho\right)^{*} K$ is an $(m, m)$-form on $M$. For every coordinate $z$, we call the function $k_{z}=\left(1_{M}, \rho\right)^{*} K / d z \wedge \overline{d z}$ on $U_{z}$ the Bergman function of $M$ relative to $z$. That is

$$
K(p, \bar{p})=k_{z}(p) d z_{p} \wedge d \bar{z}_{\bar{p}}, \quad p \in U_{z} .
$$

The Bergman functions are non-negative (cf., e.g., [2; Proposition 2.7]). It holds (cf., e.g., [2; Proposition 2.5]) that for every multi-index $A$, the $m$-form $K_{A}^{z}(p)=\partial_{A}^{\bar{z}} \cdot K(\cdot, \bar{p}) / d \bar{z}_{\bar{p}}$ belongs to $H(M)$, and that for every $\alpha \in H(M)$,

$$
\partial_{A}^{z} . \alpha(p)=\left(\alpha, K_{A}^{z}(p)\right) d z_{p} .
$$


In particular, if $A$ and $B$ are multi-indices, then

$$
\left(K_{A}^{z}(p), K_{B}^{z}(p)\right)=\partial_{B}^{z} \overline{\partial_{A}^{z}} \cdot k_{z}(p) .
$$

Let $n \in \boldsymbol{Z}_{+}$be a non-negative integer. For every $p \in M$, set

$$
H_{n}(p)=\left\{K_{A}^{z}(p) ; A \in \bigcup_{\jmath=0}^{n-1} M I(j)\right\}^{\perp} \subset H(M),
$$

where $z$ is a coordinate around $p$. The subspace $H_{n}(p)$ does not depend on the choice of $z$. Let $X \in T_{p}(M)$ be a tangent vector at $p$. For a coordinate $z$ around $p$, represent $X$ as $\left(\partial_{v}^{z}\right)_{p}$ for some $v \in C^{m}$. Then $\left(\partial_{\bar{v}}^{\bar{z}}\right)^{n}$ is a differential operator on $U_{\bar{z}}=\overline{U_{z}}$, and $K_{v}^{z}(p)=\left(\partial_{\bar{v}}^{\bar{z}}\right)^{n} \cdot K(\cdot, \bar{p}) / d \bar{z}_{\bar{p}}$ belongs to $H(M)$. Set

$$
\mu_{n}(X)=\max \left\{\left|\left(K_{v n}^{z}(p), \alpha\right)\right|^{2} ; \alpha \in H_{n}(p),\|\alpha\|=1\right\}(d z \wedge \overline{d z})_{p} .
$$

Then the $(m, m)$-form $\mu_{n}(X)$ does not depend on the representation of $X=\left(\partial_{v}^{z}\right)_{p}$ in terms of $z$ ([2; Proposition 3.7]).

We recall a lemma on a pre-Hilbert space $H$ over $\boldsymbol{C}$. We denote by $G\left(x_{1}, \cdots x_{n}\right)$ the Gramian of a system $\left(x_{1}, \cdots, x_{n}\right)$ in $H$ (especially $G(\phi)=1$ ).

LEMMA 1.1 ([2; Lemma 3.9]). Let $\left(x_{1}, \cdots, x_{n}\right)\left(n \in \boldsymbol{Z}_{+}\right)$be a linearly independent system in $H$, and let $x_{n+1} \in H$. Then the maximum of the set $\left\{\left|\left(y, x_{n+1}\right)\right|^{2} ; y \in\left\{x_{1}, \cdots, x_{n}\right\}^{\perp},\|y\|=1\right\}$ coincides with $G\left(x_{1}, \cdots, x_{n+1}\right) / G\left(x_{1}, \cdots, x_{n}\right)$.

Set $\operatorname{MII}(n)=\left\{\left(a_{1}, \cdots, a_{n}\right) \in \operatorname{MI}(n) ; a_{1} \leqq a_{2} \leqq \cdots \leqq a_{n}\right\}$. We denote by $\varphi_{n}=$ $\left(\begin{array}{c}m+n \\ n\end{array}\right)$ the cardinality of the set $\bigcup_{\jmath=0}^{n} \operatorname{MII}(j)$, and fix a numbering $\Phi$ of $\bigcup_{j=0}^{\infty} \operatorname{MII}(j)$ such that $\operatorname{MII}(n)=\left\{\Phi\left(\varphi_{n-1}+1\right), \cdots, \Phi\left(\varphi_{n}\right)\right\}$. For a sequence $\left(j_{1}, \cdots\right.$, $\left.j_{u}, s, t\right)$ of positive integers, set

$$
\left\{\begin{array}{l}
\mathcal{L}_{z}\left(j_{1}, \cdots, j_{u}\right)=\left[\partial_{\Phi(i)}^{z} \overline{\partial_{\Phi(l)}^{z}} \cdot k_{z}\right]_{l=j_{1}, \cdots, j_{u}}^{l=j_{1}, \cdots, j_{u}^{u}} \\
L_{z}\left(j_{1}, \cdots, \jmath_{u}\right)=\operatorname{det} \mathcal{L}_{z}\left(\jmath_{1}, \cdots, j_{u}\right) \quad\left(L_{z}(\phi)=1\right) \\
L_{z}\left(j_{1}, \cdots, j_{u} ; s, t\right)=\operatorname{det}\left[\partial_{\Phi(i)}^{z} \overline{\partial_{\Phi(l)}^{z}}, k_{z}\right]_{l=j_{1}, \cdots, j_{u}, \cdots, s}^{l=j_{u}, t} .
\end{array}\right.
$$

By (1.2), $\mathcal{L}_{z}\left(\jmath_{1}, \cdots, j_{u}\right)(p)$ is the transpose of the Gram matrix of the system $\left(K_{\Phi\left(j_{1}\right)}^{z}(p), \cdots, K_{\Phi\left(j_{u}\right)}^{z}(p)\right)$, and $L_{z}\left(j_{1}, \cdots, j_{u}\right)(p)$ is its Gramian.

Now, let $f_{n, z}$ be the function on $U_{z} \times C^{m}$ defined by

$$
\mu_{n}\left(\left(\partial_{v}^{z}\right)_{p}\right)=f_{n, z}(p, v)(d z \wedge \overline{d z})_{p}, \quad(p, v) \in U_{z} \times C^{m} .
$$

If $\left\{K_{\Phi\left(j_{1}\right)}^{z}(p), \cdots, K_{\left.\Phi\left(j_{u}\right)(p)\right\}}^{z}\right.$ is a maximal linearly independent subset of $\left\{K_{A}^{z}(p) ; A \in \bigcup_{\jmath=1}^{n-1} \operatorname{MII}(j)\right\}$, then Lemma 1.1, together with (1.2), implies that

$$
\begin{aligned}
& f_{n, z}(p, v)=L_{z}\left(j_{1}, \cdots, j_{u}\right)(p)^{-1} \\
& \quad \times \sum_{\varphi_{n-1}<s, t \leq \varphi_{n}} C_{\Phi(s)} C_{\Phi(t)} v^{\Phi(s)} \bar{v}^{\Phi(t)} L_{z}\left(\jmath_{1}, \cdots, \jmath_{u} ; s, t\right)(p) .
\end{aligned}
$$


Here $C_{A}=n ! / n_{1} ! \cdots n_{m} !$ and $v^{A}=v^{a_{1}} \cdots v^{a_{n}}\left(A=\left(a_{1}, \cdots, a_{n}\right) \in \operatorname{MII}(n), v=\left(v^{1}, \cdots, v^{m}\right)\right.$ $\left.\in C^{m}\right)$, where $n_{\nu}$ is the cardinality of the set $\left\{j \in\{1, \cdots, n\} ; a_{j}=\nu\right\} \quad(\nu=1, \cdots, m)$.

Proposition 1.2. The function $f_{n, z}$ is upper semi-continuous on $U_{z} \times \boldsymbol{C}^{m}$.

Proof. The proof is reduced to the following lemma.

LEMMA 1.3. Let $f$ be the function on the power $H^{n+1}$ of a pre-Hilbert space $H$ over $\boldsymbol{C}$ given by

$$
f\left(x_{1}, \cdots, x_{n+1}\right)=\max \left\{\left|\left(y, x_{n+1}\right)\right|^{2} ; y \in\left\{x_{1}, \cdots, x_{n}\right\}^{\perp},\|y\|=1\right\} .
$$

Then $f$ is upper semi-continuous on $H^{n+1}$.

Proof. Let $x^{0}=\left(x_{1}^{0}, \cdots, x_{n+1}^{0}\right) \in H^{n+1}$ be fixed, and let $\left\{x_{\sigma(1)}^{0}, \cdots, x_{\sigma(u)}^{0}\right\}$ be a maximal linearly independent subset of $\left\{x_{1}^{0}, \cdots, x_{n}^{0}\right\}$. Then $G\left(x_{\sigma(1)}, \cdots, x_{\sigma(u)}\right)$ is positive in a neighborhood of $x^{0}$. So, by Lemma 1.1 we have

as desired.

$$
\begin{aligned}
\limsup _{x \rightarrow x^{0}} f(x) & \leqq \limsup _{x \rightarrow x^{0}} \max \left\{\left|\left(y, x_{n+1}\right)\right|^{2} ; y \in\left\{x_{\sigma(1)}, \cdots, x_{\sigma(u)}\right\}^{\perp},\|y\|=1\right\} \\
& =\limsup _{x \rightarrow x^{0}} G\left(x_{\sigma(1)}, \cdots, x_{\sigma(u)}, x_{n+1}\right) / G\left(x_{\sigma(1)}, \cdots, x_{\sigma(u)}\right) \\
& =f\left(x^{0}\right),
\end{aligned}
$$

2. An intrinsic fibre pseudo-metric on the holomorphic vector bundle $S^{n} T(M)$. For $n \in Z_{+}$and $p \in M$, we consider the following condition:

$\left(C_{n}\right)_{p}$ For every non-zero vector $\left(\xi^{A}\right)_{A \in \operatorname{MII}(n)}$ of dimension $\left(\begin{array}{c}m+n-1 \\ n\end{array}\right)$, there exists $\alpha \in H_{n}(p)$ such that $\Sigma_{A} \xi^{A} \partial_{A}^{2} . \alpha(p) \neq 0$.

Condition $\left(C_{n}\right)$ stands for that $\left(C_{n}\right)_{p}$ hold for all $p \in M$. From (1.1), we reduce the following ([2; Lemma 3.4]):

Conditions $\left(C_{j}\right)_{p}(j=0, \cdots, n)$ hold if and only if the set $\left\{K_{A}^{z}(p) ; A \in \bigcup_{j=0}^{n} \operatorname{MII}(j)\right\}$ is linearly independent, or $\mathcal{L}_{z}\left(1, \cdots, \varphi_{n}\right)(p)$ is positive definite.

Now, suppose $M$ satisfies condition $\left(C_{0}\right)$. Then (1.4) implies that $\mu_{0}(X)=$ $k_{z}(p)(d z \wedge \overline{d z})_{p}$ for every $X \in T_{p}(M)$, and that $k_{z}>0$ on $U_{z}$. So, $[0,+\infty)$-valued functions $\mu_{0, n}=\mu_{n} / \mu_{0}(n \in N)$ on the holomorphic tangent bundle $T(M)$ are well defined. Every function $\mu_{0, n}$ is upper semi-continuous on $T(M)$ (by Proposition 1.2) and satisfies the following: $\mu_{0, n}(\xi X)=|\xi|^{2 n} \mu_{0, n}(X)$ for $X \in T(M)$ and $\xi \in C$; therefore $\left(\mu_{0, n}\right)^{1 / 2 n}$ is an upper semi-continuous Finsler pseudo-metric on $M$. Moreover, $\mu_{0, n}$ are biholomorphic invariants, i. e. $\mu_{0, n}(X)=\mu_{0, n}\left(f_{*} X\right), X \in T(M)$ for every biholomorphic mapping $f$ from $M$ onto another complex manifold ([2; 
Proposition 3.2]).

We denote by $S^{n} T_{p}(M)$ (resp. $S^{n} T(M)$ ) the $n$-th symmetric tensor power of $T_{p}(M)$ (resp. $T(M)$ ). $S^{n} T(M)$ is a holomorphic vector bundle over $M$, and $\left\{\partial_{A}^{z} ; A \in \operatorname{MII}(n)\right\}$ forms its local frame on $U_{z}$.

We shall show the following assertion.

THEOREM 2.1. If a complex manifold $M$ satisfies condition $\left(C_{0}\right)$, then for every $n \in N$ and $p \in M$ there exists a unique hermitian pseudo-inner-product $g^{(n)}(\cdot, \cdot)$ on the space $S^{n} T_{p}(M)$ such that

$$
(n !)^{-2} \mu_{0, n}(X)=g^{(n)}\left(X^{n}, \overline{X^{n}}\right), \quad X \in T_{p}(M),
$$

where $X^{1}=X, X^{\jmath}=X \cdot X^{\jmath-1}$ (the symmetric tensor product). Furthermore, the fibre pseudo-metric $g^{(n)}$ on $S^{n} T(M)$ is biholomorphic invariant, i.e. $g^{(n)}(Y, \bar{Y})=$ $g^{(n)}\left(f_{*} Y, \overline{f_{*} Y}\right)$ for $Y \in S^{n} T(M)$ and for any biholomorphic mapping $f$ from $M$ onto another complex manifold.

Remark 2.2. The constant $(n !)^{-2}$ in the formula (2.2) is chosen so that when $M$ is the unit disk $\{\xi \in C ;|\xi|<1\}$ in $C$ the inner product $g^{(n)}(\cdot, \cdot)$ on $S^{n} T_{0}(M)$ at the origin $0 \in M$ has the simplest form, $g^{(n)}\left(X^{n}, \overline{X^{n}}\right)=n+1$ for $X=(\partial / \partial \xi)_{0} \in$ $T_{0}(M)$ (cf. [1]).

Proof of Theorem 2.1 (Existence). Let $\left\{K_{\Phi\left(\jmath_{1}\right)}^{z}(p), \cdots, K_{\Phi\left(\jmath_{u}\right)}^{z}(p)\right\}$ be a maximal linearly independent subset of $\left\{K_{A}^{z}(p) ; A \in \bigcup_{\jmath=1}^{n-1} \operatorname{MII}(j)\right\}$. By (1.4) we have

$$
\begin{aligned}
& \mu_{0, n}\left(\left(\partial_{v}^{z}\right)_{p}\right)=L_{z}\left(\jmath_{1}, \cdots, \jmath_{u}\right)(p)^{-1} k_{z}(p)^{-1} \\
& \quad \times \sum_{\varphi_{n-1}<s, t \leq \varphi_{n}} C_{\Phi(s)} C_{\Phi(t)} v^{\Phi(s)} \bar{v}^{\Phi(t)} L_{z}\left(j_{1}, \cdots, \jmath_{u} ; s, t\right)(p) .
\end{aligned}
$$

So, the function $g^{(n)}(\cdot, \overline{)})$ defined by sesqui-bilinearity and by the requirement

$$
\begin{aligned}
& g^{(n)}\left(\left(\partial_{\Phi(s)}^{z}\right)_{p},\left(\overline{\partial_{\Phi(t)}}\right)_{p}\right) \\
& \quad=(n !)^{-2} L_{z}\left(j_{1}, \cdots, \jmath_{u}\right)(p)^{-1} k_{z}(p)^{-1} L_{z}\left(\jmath_{1}, \cdots, \jmath_{u} ; s, t\right)(p)
\end{aligned}
$$

has the desired property. Thus, the existence is proved.

To complete the proof, we prepare two lemmas.

LEMMA 2.3. Let $R=\sum_{n=0}^{\infty} R_{n}$ be a commutative, associative, graded algebra over $\boldsymbol{C}$. For every $n \in \boldsymbol{N}$, there exists a linear form $F_{n}\left(t_{0}, t_{1}, \cdots, t_{3 n-1}\right)$ on $\boldsymbol{C}^{3 n}$ such that

$$
\left(x^{n}, y^{n}\right)_{n}=F_{n}\left(f(1), f(\rho), \cdots, f\left(\rho^{3 n-1}\right)\right)
$$

for $x, y \in R_{1}$ and for any sesqui-bilinear form $(,)_{n}$ on $R_{n}$, where $\rho=e^{2 \pi \sqrt{ }-1 / 3 n}$ and $f(\xi)=f_{x, y}(\xi)=\left((x+\xi y)^{n},(x+\xi y)^{n}\right)_{n}, \quad \xi \in \boldsymbol{C}$.

Proof. Since 


$$
f\left(\rho^{l}\right)=\sum_{l, j=0}^{n}\left(\begin{array}{c}
n \\
i
\end{array}\right)\left(\begin{array}{c}
n \\
j
\end{array}\right)\left(x^{n-\imath} y^{\imath}, x^{n-\jmath} y^{j}\right)_{n} \rho^{l(i-j)},
$$

and since

$$
\sum_{l=0}^{n-1} \rho^{3 j l}= \begin{cases}n, & n \mid j \\ 0, & n \nmid j\end{cases}
$$

for every $\jmath \in \boldsymbol{Z}$, it follows that

$$
\left\{\begin{array}{l}
\sum_{l=0}^{n-1} f\left(\rho^{3 l}\right)=n(\bar{\eta}+\eta+\zeta) \\
\sum_{l=0}^{n-1} f\left(\rho^{3 l+1}\right)=n\left(\bar{\eta} \rho^{n}+\eta \rho^{-n}+\zeta\right) \\
\sum_{l=0}^{n-1} f\left(\rho^{3 l+2}\right)=n\left(\bar{\eta} \rho^{-n}+\eta \rho^{n}+\zeta\right),
\end{array}\right.
$$

where $\eta=\left(x^{n}, y^{n}\right)_{n}, \quad \zeta=\sum_{\jmath=0}^{n}\left(\begin{array}{c}n \\ j\end{array}\right)^{2}\left(x^{n-\jmath} y^{\jmath}, x^{n-\jmath} y^{j}\right)_{n}$. So, if $F^{(i)}\left(t_{0}, \cdots, t_{3 n-1}\right)=$ $\sum_{l=0}^{n-1} t_{3 l+\imath}(\imath=0,1,2)$, and $\omega=\rho^{n}=e^{2 \pi \sqrt{ }-1 / 3}$, then the form $F_{n}=\left(F^{(0)}+\omega F^{(1)}+\omega^{2} F^{(2)}\right)$ $/ 3 n$ has the desired property.

Given $n, j \in \boldsymbol{N}$ with $j \leqq n$, denote by $P_{j}^{n}$ the linear operator from $\boldsymbol{C}\left[t_{1}, \cdots, t_{j}\right]$ into $C\left[t_{1}, \cdots, t_{n}\right]$, given by $P_{j}^{n}\left(f\left(t_{1}, \cdots, t_{j}\right)\right)=\sum_{\sigma \in \Sigma(j, n)} f\left(t_{\sigma(1)}, \cdots, t_{\sigma(j)}\right), f\left(t_{1}, \cdots, t_{j}\right)$ $\in \boldsymbol{C}\left[t_{1}, \cdots, t_{j}\right]$, where $\Sigma(j, n)$ means the family of all strictly increasing mappings from $\{1, \cdots, j\}$ into $\{1, \cdots, n\}$.

LEMMA 2.4. For every $n \in \boldsymbol{N}$ it holds that

$$
n ! t_{1} \cdots t_{n}=\sum_{j=0}^{n-1}(-1)^{j} P_{n-j}^{n}\left(\left(t_{1}+\cdots+t_{n-j}\right)^{n}\right) .
$$

Proof. Let $f\left(t_{1}, \cdots, t_{n}\right)$ be the right hand side of the desired formula, and set

$$
f_{\jmath}\left(t_{1}, \cdots, t_{n}\right)=\Sigma_{\sigma \in \Sigma(n-\jmath, n)}\left(t_{\sigma(1)}+\cdots+t_{\sigma(n-j)}\right)^{n}
$$

for $\jmath=0,1, \cdots, n-1$; thus $f=\sum_{\jmath=0}^{n-1}(-1)^{\jmath} f_{\jmath}$. For every $j$,

where

$$
f_{j}\left(0, t_{2}, \cdots, t_{n}\right)=g_{j}\left(t_{2}, \cdots, t_{n}\right)+h_{j}\left(t_{2}, \cdots, t_{n}\right),
$$

$$
\left\{\begin{array}{l}
g_{j}\left(t_{2}, \cdots, t_{n}\right)=\sum_{\sigma \in \Sigma(n-\jmath, n), \sigma(1)=1}\left(t_{\sigma(2)}+\cdots+t_{\sigma(n-j)}\right)^{n} \\
h_{j}\left(t_{2}, \cdots, t_{n}\right)=\sum_{\sigma \in \Sigma(n-\jmath, n), \sigma(1) \geqq 2}\left(t_{\sigma(1)}+\cdots+t_{\sigma(n-j)}\right)^{n} .
\end{array}\right.
$$

It is easily seen that $g_{n-1}=0, h_{0}=0$, and $g_{j}=h_{j+1}(j=0,1, \cdots, n-2)$. From these we get $f\left(0, t_{2}, \cdots, t_{n}\right)=0$; therefore, the symmetry of $f$ implies $f\left(t_{1}, \cdots, t_{\jmath-1}, 0\right.$, $\left.t_{j+1}, \cdots, t_{n}\right)=0$ for any $j$. It follows from the remainder theorem that $f\left(t_{1}, \cdots, t_{n}\right)$ $=c t_{1} \cdots t_{n}$ for some constant $c$. Among expansions of $f_{0}$ into monomials the term $t_{1} \cdots t_{n}$ appears only in $f_{0}=\left(t_{1}+\cdots+t_{n}\right)^{n}$, for which the coefficient of $t_{1} \cdots t_{n}$ is $n$ !. So, the above constant must be $n$ !, as desired.

Proof of Theorem 2.1 (Uniqueness). Lemmas 2.3 and 2.4 imply that every $g^{(n)}\left(\left(\partial_{A}^{z}\right)_{p},\left(\overline{\partial_{B}^{z}}\right)_{p}\right),(A, B \in \mathrm{MI}(n))$ can be written as a linear combination of terms 
$g^{(n)}\left(X^{n}, \bar{X}^{n}\right)\left(X \in T_{p}(M)\right)$. From this we obtain the uniqueness of $g^{(n)}$. The invariant property of $g^{(n)}$ follows from the uniqueness and the invariant property of $\mu_{0, n}$ stated before. The proof is now complete.

THEOREM 2.5. Suppose $M$ satisfies conditions $\left(C_{0}\right), \cdots,\left(C_{n-1}\right)$ with $n \geqq 1$. Then $g^{(n)}$ is a differential pseudo-metric, and its components $g_{2, A \bar{B}}^{(n)}=g^{(n)}\left(\partial_{A}^{z}, \overline{\partial_{B}^{z}}\right)(A, B \in$ $\operatorname{MI}(n))$ relative to a coordinate $z$ satisfy

$$
g_{z, \Phi(s)}^{(n)} \overline{\Phi(t)}=L_{z}\left(1, \cdots, \varphi_{n-1} ; s, t\right) /\left\{(n !)^{2} k_{z} L_{z}\left(1, \cdots, \varphi_{n-1}\right)\right\}
$$

on $U_{z}$ for $s, t \in\left\{\varphi_{n-1}+1, \cdots, \varphi_{n}\right\}$. In particular, $g_{z, a \bar{b}}^{(1)}=\partial_{a}^{z} \overline{\partial_{b}^{z}} \cdot \log k_{z}$, i.e. $g^{(1)}$ is the usual Bergman pseudo-metric on $M$ ([4; pp. 271-272]).

Proof. By (2.1) the hypothesis implies that the system $\left\{K_{\Phi(1)}^{z}(p), \cdots\right.$, $\left.K_{\Phi\left(\varphi_{n-1}\right)}^{z}(p)\right\}$ itself is linearly independent for every $p \in U_{z}$. So, all the assertions follow from (2.3).

THEOREM 2.6. Suppose $M$ satisfies conditions $\left(C_{0}\right), \cdots,\left(C_{n-1}\right)$ with $n \geqq 1$. Then the pseudo-inner-product $g^{(n)}(\cdot, \cdot)$ on $S^{n} T_{p}(M)$ is an inner product if and only if condition $\left(C_{n}\right)_{p}$ holds. In particular, the fibre pseudo-metric $g^{(n)}$ is a metric if and only of condition $\left(C_{n}\right)$ holds.

Proof. Let $z$ be a coordinate around $p$. It follows from Theorem 2.5 that $g^{(n)}(\cdot, \cdot)$ is an inner product if and only if the following holds:

$$
\left\{\begin{array}{l}
\text { The matrix }\left[L_{z}\left(1, \cdots, \varphi_{n-1} ; s, t\right)\right]_{t=\varphi_{n-1}=\varphi_{n-1}, \cdots, \varphi_{n}}(p) \text { is } \\
\text { positive definite. }
\end{array}\right.
$$

If $j \in \boldsymbol{Z}$ with $j>\varphi_{n-1}$, applying Sylvester's theorem to the $(j, j)$-matrix $\mathcal{L}_{z}(1, \cdots$, $\left.\varphi_{n-1}, \cdots, j\right)$ and its minnor determinants $L_{z}\left(1, \cdots, \varphi_{n-1} ; s, t\right)\left(\varphi_{n-1}<s, t \leqq j\right)$, we have

$$
\begin{aligned}
& \operatorname{det}\left[L_{z}\left(1, \cdots, \varphi_{n-1} ; s, t\right)\right]_{t=\varphi_{n-1}}^{s=\varphi_{n-1}+\cdots, \cdots, j} \\
& =L_{z}(1, \cdots, j) L_{z}\left(1, \cdots, \varphi_{n-1}\right)^{j-\varphi_{n-1-1}} \text {. }
\end{aligned}
$$

Thereby, employing (2.1), one can see that the following four statements are mutually equivalent :

(i) Condition $\left(C_{n}\right)_{p}$ holds.

(ii) $L_{z}(1, \cdots, j)(p)>0$ for any $\jmath \in Z$ with $\varphi_{n-1}<j \leqq \varphi_{n}$.

(iii) $\operatorname{det}\left[L_{z}\left(1, \cdots, \varphi_{n-1} ; s, t\right)\right]_{t=\varphi_{n-1}=\varphi_{n-1}+1, \cdots, j}(p)>0$ for any $\jmath \in Z$ with $\varphi_{n-1}<j \leqq \varphi_{n}$.

(iv) Condition (2.4) holds.

This completes the proof of Theorem 2.6.

3. Connection of the hermitian vector bundle $\left(S^{n} T(M), g^{(n)}\right)$. If $M$ satisfies conditions $\left(C_{0}\right), \cdots,\left(C_{n}\right)$ for some $n \in N$, then, as we have seen in Theorems 2.5 and 2.6, $g^{(n)}$ is a usual hermitian fibre metric on the holomorphic vector 
bundle $S^{n} T(M)$. We shall investigate the curvature of the hermitian connection of the hermitian vector bundle $\left(S^{n} T(M), g^{(n)}\right)$ in the sense of Kobayashi and Nomizu [6; pp. 178-185] (also cf. [5; pp. 37-39]). Let $z$ be a coordinate in $U_{z} \subset M$. We denote by $\left(g_{z}^{(n) \bar{B} A}\right)_{A, B \in \operatorname{MII}(n)}$ the inverse matrix of $\left(g_{z}^{(n)}, \bar{B}\right)_{A, B \in \operatorname{MII}(n)}$ in the sense that

$$
\sum_{B \in \operatorname{MII}(n)} g_{z, A \bar{B}}^{(n)} g_{z}^{(n) \bar{B} C}=\delta_{A}^{C}, \quad A, C \in \operatorname{MII}(n) .
$$

Let $R^{(n)}$ be the curvature of the hermitian connection of $\left(S^{n} T(M), g^{(n)}\right)$, and let $R_{z, A \bar{B} \mid c \bar{d}}^{(n)}=g^{(n)}\left(R^{(n)}\left(\partial_{c}^{z}, \overline{\partial_{d}^{z}}\right) \overline{\partial_{B}^{z}}, \partial_{A}^{z}\right)$ for $A, B \in \operatorname{MI}(n)$ and $c, d \in\{1, \cdots, m\}=\operatorname{MI}(1)$. It is known $([5,6,7])$ that

$$
R_{z, A \bar{B} \mid c \bar{d}}^{(n)}=\partial_{c}^{z} \overline{\partial_{d}^{z}} \cdot g_{z, A \bar{B}}^{(n)}-\sum_{P, Q \in \operatorname{MII}(n)} g_{z}^{(n) \bar{Q} P}\left(\partial_{c}^{z} \cdot g_{z, A \bar{Q}}^{(n)}\right)\left(\overline{\partial_{d}^{z}} \cdot g_{z, P \bar{B}}^{(n)}\right) .
$$

We shall show the following.

Theorem 3.1. Suppose $M$ satisfies conditions $\left(C_{0}\right), \cdots,\left(C_{n}\right)$ with $n \in \boldsymbol{N}$. Then

$$
\begin{aligned}
R_{z, A \bar{B} \mid c \bar{d}}^{(n)}= & (n+1)^{2} g_{z, A C B \bar{d}}^{(n+1)}-g_{z, c \bar{d}}^{(1)} g_{z, A \bar{B}}^{(n)} \\
& -n^{2} \sum_{P, Q \in \operatorname{MII}(n-1)} g_{z}^{(n-1) \bar{Q} P} g_{z, P C \bar{B}}^{(n)} g_{z, A \bar{Q} \bar{d}}^{(n)}
\end{aligned}
$$

on $U_{z}$ for $A, B \in \operatorname{MI}(n)$ and $c, d \in \operatorname{MI}(1)$, where $g_{z}^{(0) \phi} \bar{\phi}=1$.

Taking $n=1$ in the above theorem we obtain the following result of Fuks $[3 ;$ p. 525$]$.

Corollary 3.2. Suppose $M$ satısfies conditions $\left(C_{0}\right)$ and $\left(C_{1}\right)$. Let $H S C(X)$ be the holomorphic sectional curvature of the Bergman metric $g^{(1)}$ on $M$ in the direction $X \in T_{p}(M)-\{0\}$, i.e.

$$
H S C(X)=-\sum_{a, b, c, d} R_{z, a \bar{b} \mid c \bar{d}}^{(1)}(p) v^{a} \bar{v}^{b} v^{c} \bar{v}^{d} / g^{(1)}(X, \bar{X})^{2},
$$

where $z$ is a coordinate around $p$ and $X=\left(\partial_{v}^{z}\right)_{p}$. Then it holds that

$$
\mu_{0,2}=(2-H S C)\left(\mu_{0,1}\right)^{2} \text { on } T(M) \text { - }\{\text { the zero section }\} \text {. }
$$

Remark 3.3. Theorem 3.1, combined with (3.2), says that when $M$ satisfies conditions $\left(C_{0}\right), \cdots,\left(C_{n}\right)$ with $n \in N$ every component of the fibre (pseudo-) metrics $g^{(2)}, \cdots, g^{(n+1)}$ is written as a rational function of the derivatives of the components of the Bergman metric $g^{(1)}$.

The remainder of this section is devoted to prove Theorem 3.1. From now on, we suppose that $M$ satisfies conditions $\left(C_{0}\right), \cdots,\left(C_{n}\right)$ for some fixed $n \in \boldsymbol{N}$. We also fix a coordinate $z$ in $U \subset M$, and suppress the dependence on $z$, i.e. $\partial_{A}=\partial_{A}^{z}, k=k_{z}, L\left(j_{1}, \cdots, j_{u}\right)=L_{z}\left(j_{1}, \cdots, j_{u}\right), g_{A \bar{B}}^{(n)}=g_{z, A \bar{B}}^{(n)}$, etc.

For every pair of multi-indices $A$ and $B$, we shall inductively define functions $L_{A \bar{B}}^{(j)}$ on $U(\jmath=0,1, \cdots, n+1)$ as follows: 


$$
\left\{\begin{array}{l}
L_{A \bar{B}}^{(0)}=\partial_{A} \overline{\partial_{B}} \cdot k \\
L_{A \bar{B}}^{(j+1)}=L_{A \bar{B}}^{(j)}-\Sigma_{C, D \in \operatorname{Mil}(j)} L^{(j) \bar{D} C} L_{C \bar{B}}^{(j)} L_{A \bar{D}}^{(j)},
\end{array}\right.
$$

where $\left(L^{(j) \bar{D} C}\right)$ is the inverse matrix of $\left(L_{A \bar{B}}^{(j)}\right)_{A, B \in \mathrm{MII}(j)}$ in the same sense as in (3.1). Non-singularity of the latter matrix is guaranteed by Lemma 3.4 below. Notice that

$$
L_{A \bar{B}}^{(j+1)}=0 \text { when } A \text { or } B \text { belongs to } \mathrm{MI}(j) .
$$

For a sequence $\left(j_{1}, \cdots, j_{u}, s, t\right)$ of positive integers, set

$$
\left\{\begin{array}{l}
\mathcal{L}^{(j)}\left(j_{1}, \cdots, j_{u}\right)=\left[L_{\Phi^{(j)}}^{(j)} \overline{\Phi(l)}\right]_{l=j_{1}, \cdots, j_{u}=j_{u}}^{l=1} \\
L^{(j)}\left(j_{1}, \cdots, j_{u}\right)=\operatorname{det} \mathcal{L}^{(j)}\left(j_{1}, \cdots, j_{u}\right) \quad\left(L^{(j)}(\phi)=1\right) \\
L^{(j)}\left(\jmath_{1}, \cdots, j_{u} ; s, t\right)=\operatorname{det}\left[L_{\Phi_{(i)}^{(j)} \overline{\Phi(l)}}\right]_{l=\jmath_{1}, \cdots, \cdots, j_{u}, s, t}^{l, s},
\end{array}\right.
$$

where $\Phi$ is the numbering of $\bigcup_{j=0}^{\infty} \operatorname{MII}(j)$ given in $\S 1$. By (1.3) we have

$$
\left\{\begin{array}{l}
\mathcal{L}^{(0)}\left(\jmath_{1}, \cdots, \jmath_{u}\right)=\mathcal{L}\left(j_{1}, \cdots, \jmath_{u}\right) \\
L^{(0)}\left(j_{1}, \cdots, j_{u}\right)=L\left(j_{1}, \cdots, \jmath_{u}\right) \\
L^{(0)}\left(j_{1}, \cdots, j_{u} ; s, t\right)=L\left(\jmath_{1}, \cdots, j_{u} ; s, t\right) .
\end{array}\right.
$$

LEMmA 3.4. If $l \in\{1, \cdots, n+1\} ; s, t \in\left\{\varphi_{l-1}+1, \cdots, \varphi_{l}\right\}$ and $\varphi_{-1}=0$, the nthe following hold:

(i) $\mathcal{L}^{(j)}\left(\varphi_{\jmath-1}+1, \cdots, \varphi_{j}\right)$ is positive-definte for every $\jmath \in\{0, \cdots, l-1\}$.

(ii) $L\left(1,2, \cdots, \varphi_{l-1}\right)=\prod_{\jmath=0}^{l-1} L^{(\jmath)}\left(\varphi_{j-1}+1, \cdots, \varphi_{j}\right)$.

(iii) $L\left(1,2, \cdots, \varphi_{l-1} ; s, t\right)=L\left(1,2, \cdots, \varphi_{l-1}\right) L_{\phi(s)}^{(l)} \overline{\Phi(t)}$.

Proof. We first recall the following well-known fact: If $A, B, C$, and $D$ are complex matrices of type $(\imath, i),(l, j),(j, i)$, and $(j, j)$, respectively, and if $A$ is non-singular, then it holds that

$$
\operatorname{det}\left[\begin{array}{ll}
A & B \\
C & D
\end{array}\right]=\operatorname{det} A \operatorname{det}\left(D-C A^{-1} B\right) \text {. }
$$

By induction on $\jmath \in\{0,1, \cdots, l-1\}$, we can show the triple assertions

$$
\begin{aligned}
& L^{(j)}\left(\varphi_{\jmath-1}+1, \cdots, r\right)>0 \quad \text { for every } \quad r \in\left\{\varphi_{\jmath-1}+1, \cdots, \varphi_{l-1}\right\}, \\
& \begin{aligned}
L\left(1,2, \cdots, \varphi_{l-1}\right)= & L^{(\jmath)}\left(\varphi_{\jmath-1}+1, \cdots, \varphi_{l-1}\right) \\
& \times \prod_{\nu=0}^{j-1} L^{(\nu)}\left(\varphi_{\nu-1}+1, \cdots, \varphi_{\nu}\right), \quad \text { and } \\
L\left(1,2, \cdots, \varphi_{l-1} ; s, t\right)= & L^{(j)}\left(\varphi_{\jmath-1}+1, \cdots, \varphi_{l-1} ; s, t\right) \\
& \times \prod_{\nu=0}^{j-1} L^{(\nu)}\left(\varphi_{\nu-1}+1, \cdots, \varphi_{\nu}\right) .
\end{aligned}
\end{aligned}
$$

In fact, assertions $(3.6)_{0},(3.7)_{0}$, and (3.8) follow from (3.4). Next, assume (3.6), 
$(3.7)_{j}$, and (3.8), hold for some $j \in\{0, \cdots, l-2\}$. Assumption (3.6), implies that $L^{(j)}\left(\varphi_{j-1}+1, \cdots, \varphi_{j}\right)>0$; therefore $L_{A \bar{B}}^{(j+1)}$ can be defined. So, by (3.5) we have

$$
L^{(\jmath)}\left(\varphi_{\jmath-1}+1, \cdots, r\right)=L^{(j)}\left(\varphi_{\jmath-1}+1, \cdots, \varphi_{j}\right) L^{(\jmath+1)}\left(\varphi_{j}+1, \cdots, r\right) .
$$

Thus, (3.6) $)_{j+1}$ and $(3.7)_{j+1}$ hold. Furthermore, if we apply (3.5) to the first matrix in the right hand side of $(3.8)_{j}$, we obtain $(3.8)_{j+1}$. The assertion (i) of Lemma 3.4 follows from (3.6), for $\jmath=0,1, \cdots, l-1$, while the assertion (ii) coincides with $(3.7)_{l-1}$. Since $L^{(l-1)}\left(\varphi_{l-2}+1, \cdots, \varphi_{l-1}\right)>0$, the assertion (iii) follows from $(3.8)_{l-1}$ and (3.5).

Proposition 3.5. For $\jmath \in\{1,2, \cdots, n+1\}$, and $A, B \in \mathrm{MI}(j)$, it holds that

$$
g_{A \bar{B}}^{(j)}=L_{A \bar{B}}^{(j)} /\left\{(\jmath !)^{2} k\right\} \text {. }
$$

Proof. Lemma 3.4 (iii) and Theorem 2.5 imply the assertion.

LEMMA 3.6. If $j \in\{1, \cdots, n\}, A, B$ are multi-indices, and $c \in \mathrm{MI}(1)$, then the following identities hold:

(i) $\partial_{c} \cdot L_{A \bar{B}}^{(j)}=L_{A C \bar{B}}^{(j)}-\sum_{P, Q \in \mathrm{MII}(\jmath-1)} L^{(\jmath-1) \bar{Q} P} L_{A \bar{Q}}^{(j-1)} L_{P C \bar{B}}^{(j)}$

(ii) $\bar{\partial}_{c} \cdot L_{A \bar{B}}^{(j)}=L_{A \overline{B C}}^{(j)}-\sum_{P, Q \in \mathrm{MII}(j-1)} L^{(j-1) \bar{Q} P} L_{A Q \bar{Q}}^{(j)} L_{P \bar{B}}^{(j-1)}$.

Proof. Identity (i) is easily shown by the definition and by induction on $\jmath$. By taking the complex conjugation of (i), we get (ii).

Proof of Theorem 3.1. Let $A, B \in \mathrm{MI}(n)$ and $c, d \in \mathrm{MI}(1)$. By applying Proposition 3.5 to the right hand side of (3.2), we get

$$
\begin{aligned}
(n !)^{2} R_{A \bar{B} \mid c \bar{d}}^{(n)}= & -\frac{1}{k^{2}} L_{A \bar{B}}^{(n)} L_{c \bar{d}}^{(1)} \\
& +\frac{1}{k}\left\{\partial_{c} \overline{\partial_{d}} \cdot L_{A \bar{B}}^{(n)}-\sum_{P, Q \in \operatorname{MI}(n)} L^{(n) \bar{Q} P}\left(\partial_{c} \cdot L_{A \bar{Q}}^{(n)}\right)\left(\overline{\partial_{d}} \cdot L_{P \bar{B}}^{(n)}\right)\right\} .
\end{aligned}
$$

Lemma 3.6, together with (3.3), implies that the term in the braces coincides with

$$
L_{A c \bar{B} d}^{(n+1)}-\sum_{P, Q \in \operatorname{MII}(n-1)} L^{(n-1) \bar{Q} P} L_{A Q \bar{d}}^{(n)} L_{P C \bar{B}}^{(n)} .
$$

So, the desired formula follows again from Proposition 3.5.

\section{REFERENCES}

[1] Azukawa, K., Square-integrable holomorphic functions on a circular domain in $\boldsymbol{C}^{n}$, Tôhoku Math. J., 39 (1985), 15-26.

[2] Azukawa, K. and J. Burbea, Hessian quartic forms and the Bergman metric, Kodai Math. J., 7 (1984), 133-152.

[3] FuKs, B. A., Ricci curvature of a Bergman metrıc invarıant under biholomorphic mappings, Soviet Math. Dokl., 7 (1966), 525-529. 
[4] Kobayashi, S., Geometry of bounded domains, Trans. Amer. Math. Soc., 92 (1959), 267-290.

[5] Kobayashi, S., Hyperbolic Manifolds and Holomorphıc Mappings, Marcel Dekker, New York, 1970.

[6] Kobayashi, S. And K. Nomizu, Foundations of Differential Geometry (vol. 2), Interscience, New York, 1969.

[7] Matsuura, S., On the theory of pseudo-conformal mappings, Sci. Rep. Tokyo Kyoiku Daigaku (A), 7 (1963), 231-253.

Department of Mathematics

TOYAMa University

TOYAMA, 930 JAPAN 